\title{
Significance of the Equilibrium Constant between Serum Ascorbate Radical and Ascorbic Acids in Man
}

\author{
Rikuro Sasaki, Teijt Kobayasi, Tadashi Kurokawa, \\ Daisuke Shibuya* and Shozo Tero-Kubota $\dagger$ \\ College of Medical Sciences, Tohoku University, *Third \\ Department of Internal Medicine, Tohoku University School \\ of Medicine, and †Chemical Research Institute of \\ Non-Aqueous Solutions, Tohoku University, Sendai 980
}

\begin{abstract}
Sasaki, R., Kobayasi, T., Kurokawa, T., Shibuya, D. and Tero-Kubota, S. Significance of the Equilibrium Constant between Serum Ascorbate Radical and Ascorbic Acids in Man. Tohoku J. exp. Med., 1984, 144 (2), 203-210— The spin concentration of ascorbate radical in human serum was examined and the existence of the stable equilibrium between the spin concentration of serum ascorbate radical and concentrations of ascorbic acids in healthy subjects was found. The value of the equilibrium constant was independent of age and sex. The administration of ascorbic acid caused the convergence of these values which were scattered within certain ranges in healthy volunteers. The values of the equilibrium constant increased in many patients with various diseases and returned to normal level with improvement of symptoms. These indicate that the equilibrium constant can be used as a clinical index to reflect metabolic state involving ascorbic acid as the radical scavenger. - spin concentration; ascorbate radical ; equilibrium constant; diabetes mellitus ; cancer ; blood diseases
\end{abstract}

It has been reported that the stable ascorbate radical [AFR] can be easily observed in human serum at room temperature, the relative electron spin resonance [ESR] intensity of AFR is proportional to the total concentration of ascorbic acid, and both the relative ESR intensity and the total concentration of ascorbic acid decline with age (Sasaki et al. 1982, 1983a). The AFR exists as the intermediate during the redox processes of the reduced form [RAsA] or the oxidized form [DAsA] of ascorbic acid, and 2 molecules of AFR disproportionate to yield RAsA and DAsA via an initial dimerization (Bielski et al. 1981). Therefore, since the radical form of ascorbic acid is the anionic one, the equilibrium among RAsA, DAsA and AFR in serum is regarded as follows:

$$
\mathrm{RAsA}+\mathrm{DAsA} \stackrel{K}{\rightleftarrows} 2 \mathrm{AFR}+\mathrm{H}^{+},
$$

where $K$ is the equilibrium constant. Thus the value of $K /\left[\mathrm{H}^{+}\right]=[\mathrm{AFR}]^{2} /$

Received for publication, February 9, 1984. 
$[\mathrm{RAsA}][\mathrm{DAsA}]$ may be calculated from each concentration of species. In the present study, the absolute concentration of $[\mathrm{AFR}]$ of serum in healthy subjects were measured and the equilibrium among RAsA, DAsA and AFR was discussed. The effects of administration of ascorbic acid were also studied. In addition, the $K /\left[\mathrm{H}^{+}\right]$value was examined in patients with various diseases.

\section{Materials and Methods}

Materials consisted of 244 healthy subjects (102 males and 142 females), whose ages ranged from 12 to 96 years, and 206 patients (122 males and 84 females), whose ages ranged from 16 to 84 years. None of the healthy subjects had supplementary vitamin intake. Blood was drawn early in the morning before breakfast. All ESR measurements were carried out with a Varian E-109E X-band EPR spectrometer at room temperature as previously reported (Sasaki et al. 1982, 1983a).

The absolute spin concentration of [AFR] was measured using an electronic double integrator of ESR (Tero-Kubota et al. 1982), for which an aqueous solution of 2, 2, 6, 6-tetramethylpiperidinoxy radical $(98 \%$, Aldrich Co.) was used as a standard. The method to determine $[\mathrm{RAsA}]$ and $[\mathrm{DAsA}]$ was described elsewhere (Sasaki et al. 1982, 1983b). In order to examine the effects of administration of ascorbic acid in healthy subjects, 47 volunteers were selected at random from 244 controls. These volunteers consisted of 21 males and 26 females, whose ages ranged from 18 to 75 years, and were given orally $1 \mathrm{~g}$ of ascorbic acid per day for one week.

Examined diseases were peptic ulcer, ulcerative colitis, hepatitis, cirrhosis of the liver and cancer of the stomach, colon, liver, gallbladder and pancreas as digestive organs, myocardial and cerebral infarction as the cardiovascular system, diabetes mellitus as metabolic disorder, leukemia, lymphoma and multiple myeloma as hematologic diseases, and bronchopneumonia as infectious disease. Most patients had specific treatment at the time of examination, except for cancer of the digestive tract. A few patients had repeated examinations.

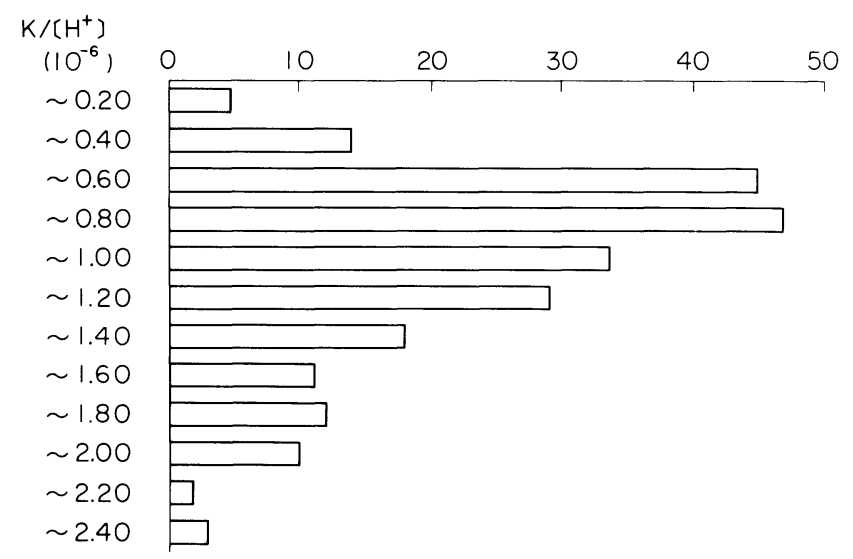

Fig. 1. Histogram of the $K /\left[\mathrm{H}^{+}\right]$value in 244 healthy subjects.

The peak was seen at $0.6-0.8 \times 10^{-6}$, though there were fairly scattered incidences over the range of $2.0 \times 10^{-6}$. 


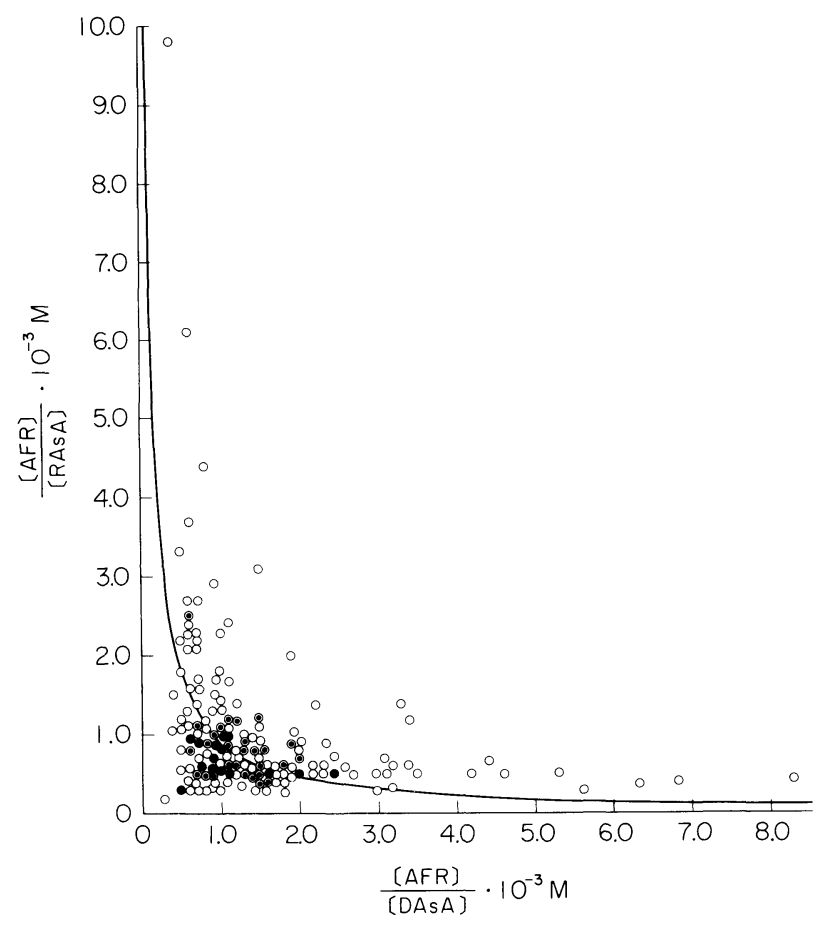

Fig. 2. Correlations between $[\mathrm{AFR}] /[\mathrm{RAsA}]$ and $[\mathrm{AFR}] /[\mathrm{DAsA}]$.

This figure clearly shows that the distribution of $(x, y)=([\mathrm{AFR}] /[\mathrm{DAsA}]$, $[\mathrm{AFR}] /[\mathrm{RAsA}])$ is centered around the curved line, $y=$ constant $/ x$, that is, the product of $[\mathrm{AFR}] /[\mathrm{RAsA}]$ by $[\mathrm{AFR}] /[\mathrm{DAsA}]$ is nearly constant. The curved line was obtained by the method of least squares. The mean $K /\left[\mathrm{H}^{+}\right]$ value calculated by the method of least squares is $0.9297 \times 10^{-6}$. This is approximated to the mean value mentioned in the text. Dotted circles ( $\odot)$ were superimposed by two, and closed ones (•) by more than three.

TABLE 1. Concentrations of ascorbic acids and AFR spin before and after administration of ascorbic acid, and statistical differences

\begin{tabular}{lcccc}
\hline & & Before & After & Student's $t$ \\
\hline RAsA & $\left(10^{-5} \mathrm{M}\right)$ & $3.69 \pm 1.28$ & $6.23 \pm 1.69$ & $8.257 \dagger$ \\
DAsA & $\left(10^{-5} \mathrm{M}\right)$ & $1.10 \pm 0.35$ & $1.34 \pm 0.35$ & $3.262^{*}$ \\
Spin $\quad\left(10^{-8} \mathrm{M}\right)$ & $1.96 \pm 0.62$ & $2.46 \pm 0.37$ & $6.493 \dagger$ \\
$K\left[\mathrm{H}^{+}\right]\left(10^{-6}\right)$ & $1.18 \pm 0.62$ & $0.80 \pm 0.25$ & $3.913 \dagger$ \\
\hline
\end{tabular}

There were statistically very significant increases in the concentrations of RAsA, DAsA and AFR spin, and very significant decrease in the $K /\left[\mathrm{H}^{+}\right]$value after administration. ${ }^{*} p<0.01$, $p<<0.001$. 


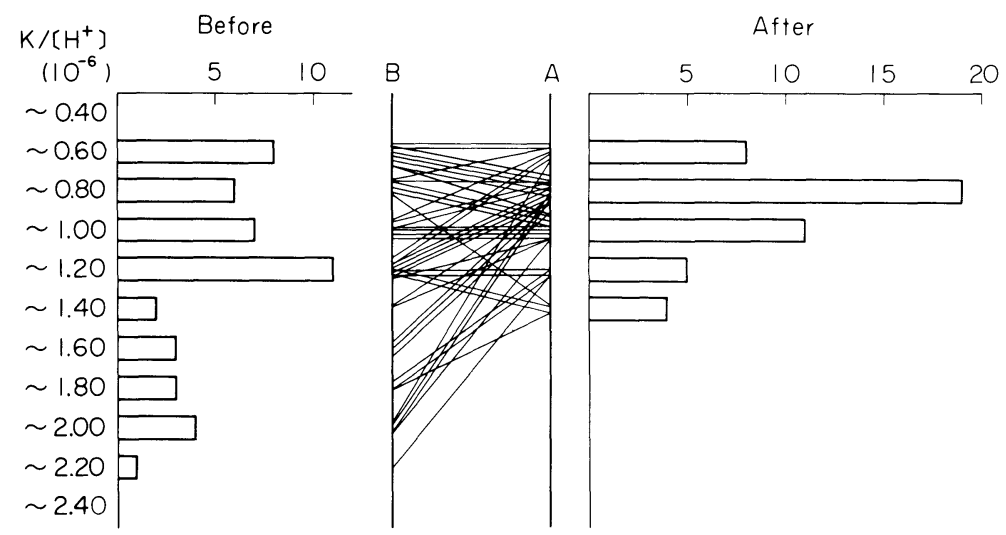

Fig. 3. Changes of incidence of the $K /\left[\mathrm{H}^{+}\right]$value after administration of ascorbic acid.

There were scattered incidences before administration but the $K /\left[\mathrm{H}^{+}\right]$values remarkably converged to $0.8 \times 10^{-6}$ after administration.

\section{RESUlts}

The spin concentration of $[\mathrm{AFR}]$ ranged from $0.33 \times 10^{-8} \mathrm{M}$ to $2.67 \times 10^{-8} \mathrm{M}$, the mean being $1.61 \times 10^{-8} \mathrm{M}$ in 244 healthy subjects. Since the mean [RAsA] and [DAsA] were $2.53 \times 10^{-5} \mathrm{M}$ and $1.48 \times 10^{-5} \mathrm{M}$, respectively, the mean spin ratio of $[\mathrm{AFR}]$ in serum was calculated to be $0.06 \%$ of the former and $0.11 \%$ of the latter. The value of $K /\left[\mathrm{H}^{+}\right]=[\mathrm{AFR}]^{2} /[\mathrm{RAsA}][\mathrm{DAsA}]$ was calculated in each subject. It ranged from $0.18 \times 10^{-6}$ to $4.85 \times 10^{-6}$, the mean being $1.078 \times$ $10^{-6}$. The histogram of the value $K /\left[\mathrm{H}^{+}\right]$is shown in Fig. 1 . The most frequent incidence of this value was seen at $0.6-0.8 \times 10^{-6}$.

Correlations between $[\mathrm{AFR}] /[\mathrm{RAsA}]$ and $[\mathrm{AFR}] /[\mathrm{DAsA}]$ are shown in Fig. 2. This figure indicates that the $K /\left[\mathrm{H}^{+}\right]$value is determined by two factors, $[\mathrm{AFR}] /[\mathrm{RAsA}]$ and $[\mathrm{AFR}] /[\mathrm{DAsA}]$, and is nearly constant. There were no correlations between this value and age or sex. While the administration of ascorbic acid caused significant increase in the concentrations of RAsA, DAsA and AFR (Table 1), the remarkable convergence of the $K /\left[\mathrm{H}^{+}\right]$was observed as shown in Fig. 3.

The spin concentration of $[\mathrm{AFR}]$ in 206 patients ranged from $0.47 \times 10^{-8} \mathrm{M}$ to $80.58 \times 10^{-8} \mathrm{M}$, the mean being $2.60 \times 10^{-8} \mathrm{M}$. The mean concentration of [RAsA ] and [DAsA] were $3.02 \times 10^{-5} \mathrm{M}$ and $1.53 \times 10^{-5} \mathrm{M}$, respectively, and the mean spin ratio was $0.08 \%$ of the former and $0.16 \%$ of the latter. The $K /\left[\mathrm{H}^{+}\right]$ value ranged from $0.08 \times 10^{-6}$ to $111.17 \times 10^{-6}$, the mean being $4.82 \times 10^{-6}$. Changes in the $K /\left[\mathrm{H}^{+}\right]$value in patients are shown in Figs. 4 and 5. The value increased in a considerable number of cases of diseases of digestive organs except for liver diseases, such as hepatitis and cirrhosis of the liver. While the value remained in the normal range in the majority of early stomach cancer, it increased 


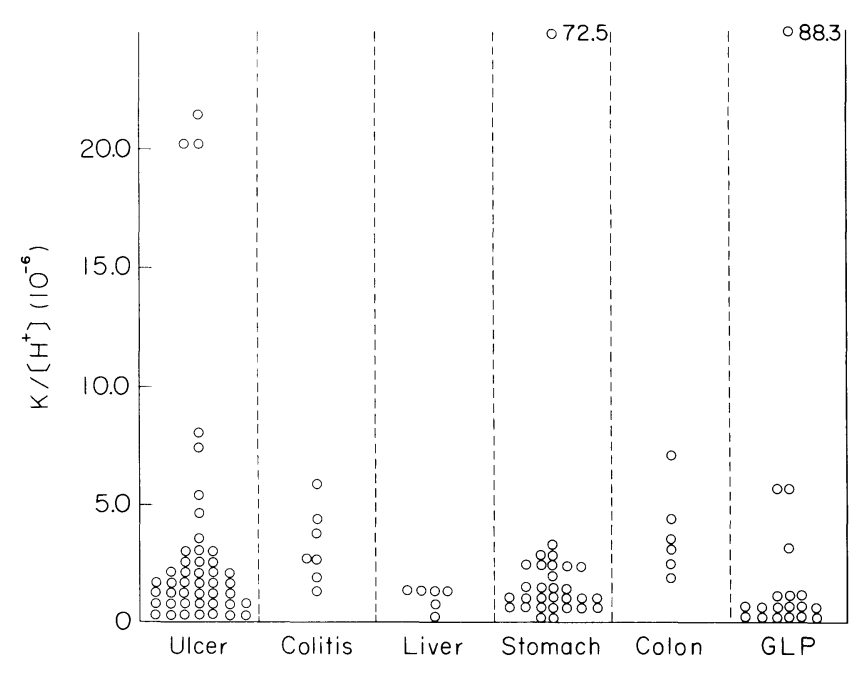

Fig. 4

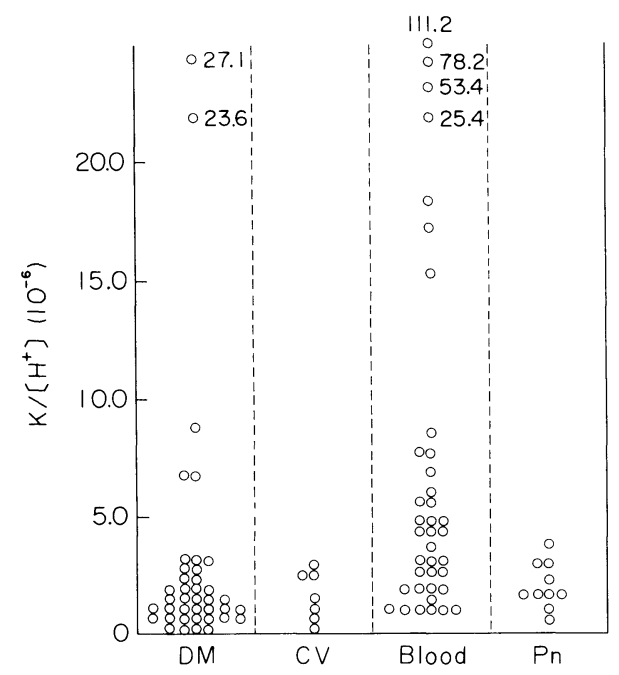

Fig. 5

Figs. 4 and 5. Changes of the $K /\left[\mathrm{H}^{+}\right]$value in patients with various diseases. Ulcer : peptic ulcer; Colitis : ulcerative colitis ; Liver : hepatitis and cirrhosis of the liver; Stomach: stomach cancer; Colon: cancer of the colon; GLP : cancer of the gallbladder, liver and pancreas; DM : diabetes mellitus ; $\mathrm{CV}$ : cerebral and myocardial infarction; Blood: leukemia, lymphoma and multiple myeloma; Pn : bronchopneumonia.

in most cases of cancer of the colon. It also increased in some cases of cancer of the liver, gallbladder and pancreas, but decreased significantly when associated with obstructive jaundice. Some of diabetics showed a remarkable increase in the $K /\left[\mathrm{H}^{+}\right]$value. The value ranged within normal limits in cases of cardiovascular diseases. It was very striking that the value increased remarkably in most cases 


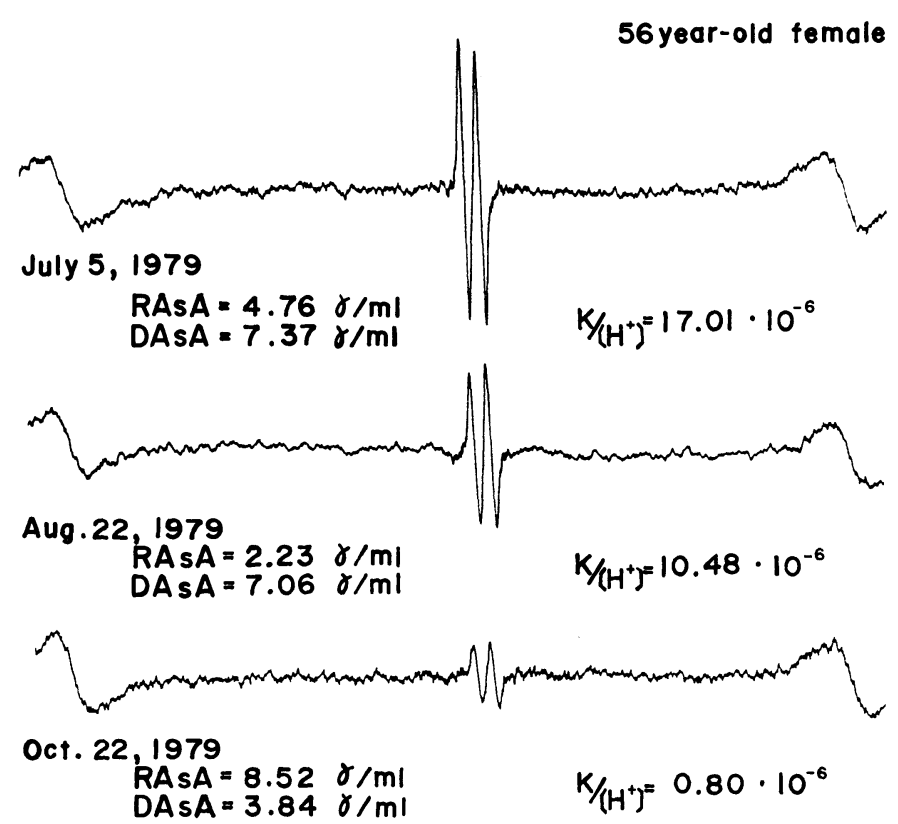

Fig. 6. Changes of ascorbate radical, ascorbic acids and the $K /\left[\mathrm{H}^{+}\right]$value in a 56 year-old patient with acute myelogenous leukemia.

The $K /\left[\mathrm{H}^{+}\right]$value was remarkably high at admission and gradually returned to normal with remssion.

of blood diseases. Fig. 5 shows that the $K /\left[\mathrm{H}^{+}\right]$value in a leukemic patient returned to the normal level when the remission was obtained. In a half of patients with bronchopneumonia, the value showed a slight increase.

\section{Discussion}

Foerster et al. (1965) have reported that the AFR is observed when ascorbate solution $\left(10^{-2}-10^{-3} \mathrm{M}\right)$ and dehydroascorbic acid solution $\left(10^{-2}-10^{-3} \mathrm{M}\right)$ were mixed, and the equilibrium constant ranges from 4.4 to $5.4 \times 10^{-9}$ at $\mathrm{pH} 6.4,25^{\circ} \mathrm{C}$, and the lower the $\mathrm{pH}$, the smaller the equilibrium constant. Though they have regarded the AFR as the neutral radical form, ESR studies have revealed the radical form of ascorbic acid is the anionic one over the $\mathrm{pH}$ range 1-12 and protonates only at still lower $\mathrm{pH}$ (Laroff et al. 1972). Accordingly, the equilibrium constant of Foerster et al. should correspond to $K /\left[\mathrm{H}^{+}\right]$. Although this value is $\mathrm{pH}$-dependent, $\left[\mathrm{H}^{+}\right]$of the $K /\left[\mathrm{H}^{+}\right]$value may be disregarded as far as the value in human serum is concerned, because the $\mathrm{pH}$ of blood is maintained at $7.40 \pm 0.05$. Hereafter the $K /\left[\mathrm{H}^{+}\right]$will be simply denoted as $K$ value.

The concentration of ascorbic acid in human serum is in the range of $10^{-5} \mathrm{M}$, which is far lower than the concentration of Foerster's experiment. The $K$ value in human serum, however, is exceedingly larger than in aqueous solutions. This difference may be partly due to the difference of $\mathrm{pH}$ and partly due to the shift of the equilibrium to the radical side in serum. While the ESR intensity of AFR 
is proportional to [RAsA] and the latter is influenced by age and sex (Loh and Wilson 1971; Bates et al. 1979 ; Sasaki et al. 1983a, b), the $K$ value is nearly constant and is independent of age or sex. These indicate the existence of stable equilibrium among RAsA, DAsA and AFR in serum in healthy subjects. This is also proved by interesting results that the value converges to the certain point when ascorbic acid is sufficiently administered. It may well be said that this point is to be the physiological $K$ value. These facts suggest that it is of great importance to examine not only the concentrations of ascorbic acids but also the equilibrium constant among RAsA, DAsA and AFR in serum.

Ascorbic acid quenches oxygen radicals (Nishikimi 1975; Fessenden and Verma 1978; Bodannes and Chan 1979) and becomes AFR. It is presumed that the AFR increases, resulting in the increase of $K$ value, under conditions in which excess free radicals are generated. The authors have observed that the $K$ value increased in a considerable percentage of patients with various diseases, and it returns to normal level with improvement of symptoms. It appears that changes in $K$ value are remarkable, when morbid symptoms are generalized. Changes in this value do not seem to specify onset or progress of particular diseases but vary depending upon stages of diseases. Therefore, this value is thought to be a clinical index to reflect metabolic state involving ascorbic acid as the radical scavenger.

Lohmann et al. (1979a, b) and Bensch et al. (1981) have reported characteristically shaped ESR signal in lyophilized blood of leukemic patients and also in the erythrocytes of patients with acute lymphatic leukemia, and that their signal decreased or disappeared with improvement of the disease. They finally attributed this signal to ascorbic acid, reacting with copper protein. It has been reported that free radicals are produced by a reaction between oxygen and a variety of lyophilized biological materials, and that ascorbic acid reacts with oxygen in the dry state only when mixed before lyophilization with a polymer such as protein (Heckly 1976). It may be certain that their signal is originated from ascorbic acid. At present, however, it is impossible to have quantitative estimation of ascorbate radical in lyophilized materials. It is thought that our simple technique to observe AFR in serum as it is and application of measuring the absolute spin concentration of AFR in normals and patients have brought fruitful information.

\section{Acknowledgments}

We thank Prof. Yusaku Ikegami for stimulating discussion and Miss Hisae Ueda for technical help.

\section{References}

1) Bates, C.J., Burr, M.K. \& StLeger, A.S. (1979) Vitamin C, high density lipoproteins and heart disease in elderly subjects. Age and Ageing, 8, 177-182. 
2) Bensch, K.G., Koerner, O. \& Lohmann, W. (1981) On a possible mechanism of action of ascorbic acid: formation of ionic bonds with biological molecules. Biochem. biophys. Res. Commun., 101, 312-316.

3) Bielski, B.H.J., Allen, A.O. \& Schwarz, H.A. (1981) Mechanism of disproportionation of ascorbate radicals. J. Amer. chem. Soc., 103, 3516-3518.

4) Bodannes, R.S. \& Chan, P.C. (1979) Ascorbic acid as a scavenger of singlet oxygen. Fed. Europ. biochem. Soc. Letters, 105, 195-196.

5) Fessenden, R.W. \& Verma, N.C. (1978) A time resolved electron spin resonance study of the oxidation of ascorbic acid by hydroxyl radical. Biophys. J., 24, 93-101.

6) Foerster, G.v., Weis, W. \& Staudinger, H. (1965) Messung der Elektronspinresonanz an Semidehydroascorbinsäure. Ann. Chem., 690, 166-169.

7) Heckly, R.J. (1976) Free radicals in dry biological systems. In: Free Radicals in Biology, edited by William A. Pryor, Vol. 2. Academic Press, New York-London, pp. $135-158$.

8) Laroff, G.P., Fessenden, R.W. \& Schuler, R.H. (1972) The electron spin resonance spectra of radical intermediates in the oxidation of ascorbic acid and related substances. J. Amer. chem. Soc., 94, 9062-9073.

9) Loh, H.S. \& Wilson, C.W.M. (1971) The relationship between leucocyte ascorbic acid and haemoglobin levels at different ages. Int. J. Vitamin Nutr. Res., 41, 259-467.

10) Lohmann, W., Schreiber, J., Gerhardt, H., Breithaupt, H., Löffler, H. \& Pralle, H. (1979a) Electron spin resonance (ESR) investigations on blood of patients with leukemia. Blut, 39, 147-151.

11) Lohmann, W., Schreiber, J., Strobelt, W. \& Müller-Eckhardt, Ch. (1979b) On the possible involvement of ascorbic acid and copper proteins in leukemia: 1. Electron spin resonance (ESR) investigations on native blood, erythrocytes, and leucocytes. Blut, 39, 317-326.

12) Nishikimi, M. (1975) Oxidation of ascorbic acid with superoxide anion generated by the xanthine-xanthine oxidase system. Biochem. biophys. Res. Commun., 53, 463 -468 .

13) Sasaki, R., Kurokawa, T. \& Tero-Kubota, S. (1982) Nature of serum ascorbate radical and its quantitative estimation. Tohoku J. exp. Med., 136, 113-119.

14) Sasaki, R., Kurokawa, T. \& Tero-Kubota, S. (1983a) Ascorbate radical and ascorbic acid level in human serum and age. J. Gerontol., 38, 26-30.

15) Sasaki, R., Kurokawa, T., Kobayasi, T. \& Tero-Kubota, S. (1983b) Influences of sex and age on serum ascorbic acid. Tohoku J. exp. Med., 140, 97-104.

16) Tero-Kubota, S., Sano, Y. \& Ikegami, Y. (1982) Monomer-dimer equilibrium of the 1-methyl-2-methoxycarbonyl-pyridinyl radical in 2-methyl-tetrahydrofuran solution. Kinetic and thermodynamic studies by electron spin resonance spectroscopy. $J$. Amer. chem. Soc., 104, 3711-3714. 